\title{
OPTIMIZED MAXIMUM LOADABILITY OF POWER SYSTEMS USING AN ENHANCED DYNAMIC JAYA ALGORITHM
}

\author{
Ibrahim A. Farhat, \\ Electrical and Computer Engineering Department, \\ Faculty of Engineering, Al-Asmarya University, \\ Libya. \\ ibrahimfarhat@yahoo.com \\ Abdullah O. Hawal, \\ Electrical and Computer Engineering Department, \\ Faculty of Engineering, Elmergib University, \\ Libya. \\ aohawal@elmergib.edu.ly
}

Submitted: Jun, 15, 2020 Revised: Aug, 29, 2020 Accepted: Sep,12, 2020

\begin{abstract}
The problem of Maximum Loadability of power systems is addressed in this paper using a proposed dynamic JAYA algorithm. The maximum loadability problem is a typical optimization problem in which the maximum loadability point is to be determined optimally. Voltage stability of power systems is maintained by determining the estimated margin between the system operating point and the maximum loadability limit. The basic JAYA algorithm has been introduced to solve foremost optimization problems with small-scaled nature. However, when applied to large-scale, nonlinear and nonconvex constrained problems, it showed a poor convergence characteristic. In order to deal with these weaknesses, the original algorithm has been improved by adding some dynamic features to its convergence behavior. The modified algorithm has been presented and validated when applied to well-known typical power systems. The obtained results were compared to the results achieved by other equivalent optimization techniques.
\end{abstract}

Keywords: JAYA algorithm, maximum loadability limit, power system optimization, voltage stability.

\section{INTRODUCTION}

Voltage instability is one of the common operation problems accompanying power system networks as a result of various operating conditions. To deal with this issue and maintain voltage stability after the occurrence of disturbances in the system, the state of equilibrium has to be restored successively. One of the most effective reasons behind the voltage instability is the extensively stressed and heavily loaded systems. Shortage of reactive power supply that does not satisfy the demand is another possible reason. Switching problems, unscheduled outages in addition to poor system voltage profiles can also cause voltage instability and lead the system to lose equilibrium.

Voltage collapse can be experienced in different parts of the network [1]. Although they are linked to each other, maximum loadability and voltage stability concepts should not be mixed up. One should think of maximum loadability problem considering the system static characteristics and not limited by the voltage stability aspects. Nevertheless, the closer the system operating point to the 
maximum loadability limit, the more likely the voltage instability to occur [2]. Figure 1 shows the P-V curve demonstrating the nose point of the maximum loading point.

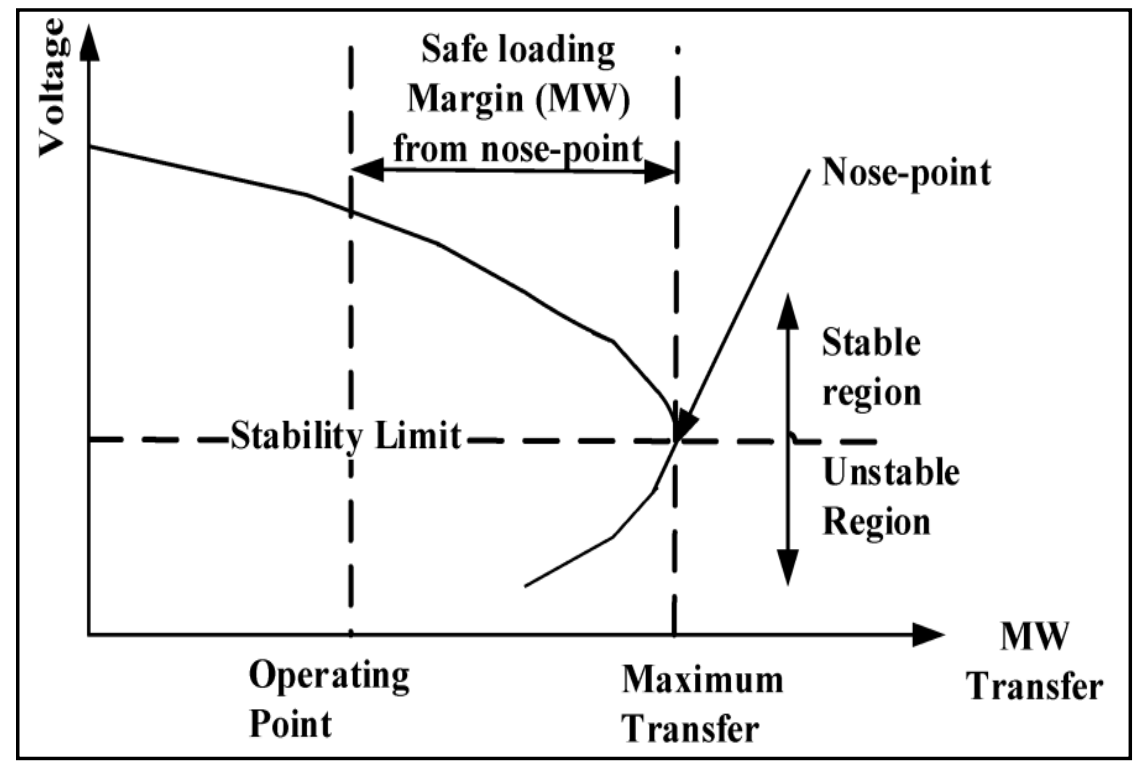

Fig. 1 P-V curve and maximum loadability limit

As shown in Figure 1, the system operates in the upper part of the curve with static and dynamic stability characteristics are feasible. In this region, the curve has a high voltage-low current profile. The maximum loadability limit point defines the voltage collapse point behind which the system loses equilibrium [1]. Optimization methods in general can be classified as classic deterministic and heuristic non-deterministic techniques. Many of these have been applied to solve the maximum loadability optimization problem.

The deterministic calculus-based approaches are the Interior Point method and the Sequential Quadratic Programing algorithms [3, 4]. More recent heuristic methods have also been utilized successfully to solve this problem. These include Particle Swarm Optimization (PSO) [5], Ant Colony Optimization (ACO) [6] and Genetic Algorithm (GA) [7].

The JAYA algorithm is one of the heuristic non-calculus-based approaches that have been applied for optimization problems. This algorithm has recently introduced as a simple and non-deterministic optimization technique [8]. In spite of its popularity and simplicity due to the limited number of parameters it requires, the basic JAYA algorithm suffers from significant convergence issues. This poor convergence behavior can be observed when applying the basic algorithm to nonlinear, non-smooth high-scaled optimization problems with nonlinear constraints. The major weakness is the divergence to local minima instead of converging to the global. It was observed through the experience and trials that some population diversity preservation issues were the reason behind this deficiency.

In this paper, a modified adaptive JAYA algorithm, MAJAYA, is presented and applied to determine the maximum loadability limit of power systems. The reminder of the paper is organized as follows: Section 2 provides the formulation of the problem. In Section 3, the MAJAYA is described. Simulation results are demonstrated in Section 4. The conclusion is drawn in Section 5. 


\section{MAXIMUM LOADABILITY}

The optimization problem of the Maximum Loadability is formulated as a non-linear constrained optimization problem [3].

\section{A. Objective Function}

The objective of the problem is to find the maximum loading of a power system taking into consideration the operational constrains of the system [3]. The maximum loadability problem is formulated as follows:

$$
\operatorname{Max} f=\lambda
$$

where $\lambda$ is the load incremental parameter with reference to the current operating point of the system. This parameter is bounded by its initial value which is $O$ and its upper limit $\lambda$ collapse at the voltage collapse point. Accordingly, the power demand of the buses increases instantaneously as follows:

$$
\begin{gathered}
P_{d i}=P_{d i 0}+\lambda P_{d} \\
Q_{d i}=Q_{d i 0}+\lambda Q_{d}
\end{gathered}
$$

where $P_{d i}$ and $Q_{d i}$ are the active and reactive power at the $i^{\text {th }}$ load bus, while $P_{d i o}$ and $Q_{d i o}$ are the initial active and reactive power at the $i^{\text {th }}$ load bus.

\section{B. Constraints}

The system constraints include bus voltage magnitude limits, power generation upper and lower limits, switchable capacitor limits and transformer tap changer limits.

- Load balance

$$
\begin{gathered}
P_{g i}-P_{d i}=\left|V_{i}\right| \sum_{i=1}^{N}\left|Y_{i j}\right|\left|V_{j}\right| \cos \left(\left[\delta_{i}-\delta_{j}-\theta_{i j}\right)\right. \\
Q_{g i}-Q_{d i}=\left|V_{i}\right| \sum_{i=1}^{N}\left|Y_{i j}\right|\left|V_{j}\right| \sin \left(\delta_{i}-\delta_{j}-\theta_{i j}\right)
\end{gathered}
$$

where the number of buses is $N$ and the voltage profile of the $i^{\text {th }}$ bus is $\left|V_{i}\right|$ and $\delta_{i}$ while the $i^{\text {th }}$ element of the system's $Y_{b u s}$ matrix is $\left|Y_{i j}\right|$ and $\theta_{i j}$. Power generation upper and lower limits

$$
\begin{aligned}
& P_{g i, \min } \leq P_{g i} \leq P_{g i, \max } \\
& Q_{g i, \min } \leq Q_{g i} \leq Q_{g i, \max }
\end{aligned}
$$

- Bus voltage magnitude and angle limits

$$
\begin{gathered}
|V|_{\min } \leq|V|_{i} \leq|V|_{\max } \\
\delta_{i, \min } \leq \delta_{i} \leq \delta_{i, \max }
\end{gathered}
$$

- Transformer tap changer limits 


$$
T_{k, \min } \leq T_{k} \leq T_{k, \max }
$$

- Switchable capacitor limits

$$
Q_{c i, \min } \leq Q_{c i} \leq Q_{c i, \max }
$$

In the above equations; the number of generating units is $\mathrm{Ng}$, the number $N_{b}$ is the number of system buses, $N_{t}$ is the number of tap changing transformers and $N_{s c}$ is the number of switchable shunt capacitors.

\section{THE JAYA ALGORITHM}

This section is divided into two parts. In the first the basic JAYA is explained. The modified JAYA algorithm is demonstrated in the second part.

\section{A. The Basic JAYA Algorithm}

The original JAYA algorithm is a deterministic heuristic optimization method that was introduced by R. Venkata [8] recently. JAYA is a Sanskrit word which means victory. This approach has been effectively applied to solve a number of constrained optimization problems. The very limited number of control parameters required for this algorithm is one of its good features. A short explanation on how the JAYA algorithm can be implemented is shown as follows:

1- The population size, number of variables for decision and stopping criteria are defined and the process is initialized.

2- Population of size $\mathrm{P}$ (candidate solution) $\times \mathrm{q}$ (decision variables) is generated.

3- An initial solution is determined.

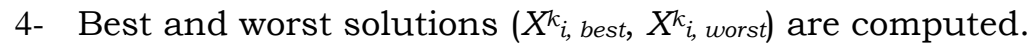

5- The solution vector is updated as follows:

$$
X_{i, j}^{k+1}=X_{i, j}^{k}+r_{1}\left(X_{i, \text { best }}^{k}-\left|X_{i, j}^{k}\right|\right)-r_{2}\left(X_{i, \text { worst }}^{k}-\left|X_{i, j}^{k}\right|\right)
$$

where, $r_{1}$ and $r_{2}$ are random numbers within the interval $[0,1]$.

6- Solution candidates are compared to check if the updated solution is better than the previous on or not. The update is accepted if the new candidate is better, otherwise it is rejected.

7- The stopping criterion is checked and applied so that the algorithm is terminated when satisfied or otherwise return to update step 2.

The JAYA algorithm requires identifying only the maximum iteration in addition to the size of population. It can also be seen from Equation (12) that the candidate moves closer to the best solution and pushes away from the worst one.

\section{B. The Modified Adaptive JAYA Algorithm}

In order to deal with the hitches linked to the basic algorithm, some changes are suggested in this section. These modifications are introduced to improve thee algorithm's behaviour when applied for large nonlinear non-smooth objective functions. 
The proposed adjustment is to dynamically update the population size. Accordingly, once the initial population size is chosen, then the population size is updated adaptively according to the following mechanism [9]:

$$
P_{\text {new }}=P_{\text {old }} \times \operatorname{round}(1+s)
$$

In the above equation $s$ is an arbitrary variable such that $-1<\mathrm{s}<1$. Consequently, in the modified adaptive algorithm (MAJAYA), all the population elements will be updated to the next population vector if the new size is larger than the old one and the optimal solution will be assigned to the remaining candidates. On the other hand, only the best solution will be moved to the next population if the old size is larger than the new one. Logically, no action is needed if there is no change in the size.

\section{SIMULATION RESULTS}

The proposed MAJAYA was employed to determine the maximum (optimal) loadability for two well-known IEEE 30 bus and IEEE 118 bus power systems. The algorithm was implemented and coded in MATLAB and executed on an Intel Core i7 $-8750 \mathrm{H} 2.20 \mathrm{GHz}$ personal computer with 8 GB RAM. In order to check for consistency, 50 independent runs were conducted with different random initial solution for each run. Results obtained were compared with those of some other methods. The various algorithm parameters were tuned independently since they were problem-dependent. A considerable number of preliminary runs were executed individually so that the optimal parameter combination was obtained.

The IEEE 30 bus system consists of six generating units, four transformers and 41 branches. The system configuration can be found in [10]. Results obtained for maximum bus voltage amplitude and angle at the maximum loading point are shown in Table 1.

Table 1: 30 bus system: Bus voltages at the maximum loading.

\begin{tabular}{|c|c|c|c|c|c|}
\hline \multirow{2}{*}{ Bus No. } & \multicolumn{2}{|c|}{ Bus Voltage } & \multirow{2}{*}{ Bus No. } & \multicolumn{2}{|c|}{ Bus Voltage } \\
\cline { 2 - 3 } & $|\boldsymbol{V}| \boldsymbol{p u} \boldsymbol{~}$ & $\boldsymbol{\delta}^{\circ}$ & & $|\boldsymbol{V}| \boldsymbol{p u}$ & $\boldsymbol{\delta}^{\circ}$ \\
\hline 1 & 1.0000 & 0.0000 & 16 & 0.9888 & -12.0034 \\
\hline 2 & 0.9998 & -3.4534 & 17 & 0.9829 & -12.7768 \\
\hline 3 & 0.9758 & -4.5678 & 18 & 0.9890 & -12.8876 \\
\hline 4 & 0.9897 & -4.7856 & 19 & 0.9756 & -12.8899 \\
\hline 5 & 0.9876 & -5.8783 & 20 & 0.9987 & -12.8987 \\
\hline 6 & 0.9843 & -5.9723 & 21 & 0.9991 & -12.6589 \\
\hline 7 & 0.9887 & -7.7685 & 22 & 0.9978 & -13.0000 \\
\hline 8 & 0.9954 & -8.3334 & 23 & 0.8936 & -13.1432 \\
\hline 9 & 0.9865 & -9.7683 & 24 & 0.9778 & -12.8798 \\
\hline 10 & 1.0000 & -10.8721 & 25 & 0.9878 & -11.5678 \\
\hline 11 & 0.9788 & -10.9867 & 26 & 0.9788 & -11.5453 \\
\hline 12 & 0.9878 & -10.9999 & 27 & 1.0000 & -11.3485 \\
\hline 13 & 0.9877 & -11.4638 & 28 & 0.9789 & -11.5456 \\
\hline 14 & 0.9856 & -11.4537 & 29 & 1.0000 & -12.7776 \\
\hline 15 & 0.9867 & -11.6665 & 30 & 0.9819 & -12.6799 \\
\hline
\end{tabular}

The proposed algorithm was once again applied to the IEEE 118 bus test system to confirm its effectiveness. The diagram of the testing system can be 
found in [10]. This system is made up of 54 generation buses, 64 load buses, and 186 branches. It also has 9 transformers and 14 switchable capacitor banks.

The results were compared to those obtained by some other evolutionary techniques previously employed to solve the problem. These are Differential Evolution (DE) [11], Multi Agent-based Hybrid Particle Swarm Optimization (MAHPSO) [12], Cuckoo Search Algorithm (CSA) [13] and Dynamic Modified Bacterial Foraging Algorithm (DMBFA) [14]. The comparison is demonstrated in Table 2.

Table 2: Comparison of the results

\begin{tabular}{|c|c|c|}
\hline \multirow{2}{*}{ Method } & \multicolumn{2}{|c|}{ Maximum power $(\mathbf{p u})$} \\
\cline { 2 - 3 } & 30-bus system & $\mathbf{1 1 8 - b u s ~ s y s t e m ~}$ \\
\hline MAHPSO & 2.6081 & 56.45 \\
\hline DE & 2.6709 & 56.543 \\
\hline CSA & 2.8396 & 62.5671 \\
\hline DMBFA & 2.9284 & 62.9865 \\
\hline MAJAYA & 2.9307 & 62.9888 \\
\hline
\end{tabular}

\section{CONCLUSIONS}

In this paper, a modified adaptive JAYA algorithm was applied to determine the maximum loadability of power systems. This problem was addressed as an optimization problem and formulated as so. The system's operation constraints were taken into account. These included voltage magnitude limits, power generation upper and lower limits, switchable capacitor limits and transformer tap changer limits. The adaptive algorithm was modified to update the solution vector and enhance the convergence properties of the algorithm. Simulation results demonstrated the robustness and effectiveness of the algorithm for the maximum loadability problem. The algorithm was tested and applied to two well-known IEEE power systems. Comparison with some selected heuristic optimization methods showed that the applied algorithm has accomplished good and satisfying results compared to those obtained by the other techniques.

\section{REFERENCES}

[1] P. Kundur, "Power System Stability and Control", McGraw -Hill, 1994.

[2] K. R. Padiyar, "Power System Dynamics: Stability and Control", BSP, $2^{\text {nd }}$ ed., 2008.

[3] G. D. Irisarri, X. Wang, J. Tong, S. Moktari, "Maximum loadability of power systems using interior point non-linear optimization method," IEEE Transactions on Power Systems, Vol. 12, No. 1, 1997, pp. 162-172.

[4] V. Ajjarapu and C. Christy, "The continuation power flow: A tool for steady state voltage stability analysis", IEEE Transactions on Power Systems, Vol. 7, No. 1, 1992, pp. 416-423.

[5] L. D. Arya, S. C. Choube, M. Shrivastava, and D. P. Kothari, "Loadability margin enhancement using coordinated aggregation based particle swarm optimization (CAPSO)" International Journal of Electrical Power Energy Systems, Vol. 32, No. 9, 2010, pp. 975-984. 
[6] M. R. Kalil, I. Musirin, and M. M. Othman, "Ant colony optimization for maximum loadability search in voltage control study," IEEE International Conference on Power and Energy, 2006, pp. 240 - 245.

[7] P. Acharjee, "Identification of Maximum Loadability Limit and Weak Buses using Security Constraint Genetic Algorithm", International Journal of Electrical Power and Energy Systems, Vol. 36, pp. 40-50, 2012.

[8] R. Venkata Rao, "Jaya: A simple and new optimization algorithm for solving constrained and unconstrained optimization problems," International journal of industrial computations, Vol. 7, pp. 19-34, 2016. 